\title{
Ensayo:
}

\section{Alejandra Pizarnik, su aporte en la construcción del concepto de mujer en el feminismo interseccional}

\author{
Fecha de recepción: 2021-10-18 • Fecha de aceptación: 2021-11-22 • Fecha de publicación: 2022-01-10
}

Rosa Estefanía Navas Espinosa

English Ride, Ecuador

englishridec@gmail.com

https://orcid.org/0000-0003-4360-769X

\section{Resumen}

El presente estudio recopila y describe la construcción del concepto de la mujer desde los aportes literarios de Pizarnik. Asimismo, se articulan escritos de Pizarnik con el enfoque del feminismo interseccional. La metodología aplicada es de tipo descriptivo-cualitativo, con un diseño narrativo. El ensayo tiene como principal objetivo describir desde varios escritos de Pizarnik, tales como poesía, diarios y citas, la construcción del concepto de la mujer, relacionando con el feminismo interseccional. En varios poemas y escritos se vislumbra varias temáticas: la noción de mujeres unidas, la ruptura de los estereotipos de género, la soledad, independencia de una mujer, el apropiarse del cuerpo, la construcción de ser mujer desde una perspectiva "outsider", las barreras infranqueables entre los derechos que poseen las mujeres y los hombres, además de la insuficiencia del lenguaje en la descripción de versiones de realidad.

\section{Palabras clave: Alejandra Pizarnik, poeta, mujer, feminismo}

\begin{abstract}
This study compiles and describes the construction of the concept of woman from Pizarnik's literary contributions. It also articulates Pizarnik's writings with the approach of intersectional feminism. The
\end{abstract}


methodology applied is descriptive-qualitative, with a narrative design. The main objective of the essay is to describe from several writings of Pizarnik, such as poetry, diaries and quotations, the construction of the concept of woman, relating it to intersectional feminism. In several poems and writings several themes are glimpsed: the notion of united women, the rupture of gender stereotypes, the loneliness, independence of a woman, the appropriation of the body, the construction of being a woman from an "outsider" perspective, the insurmountable barriers between the rights that women and men have, as well as the inadequacy of language in the description of versions of reality.

Keywords: Alejandra Pizarnik, poet, woman, feminism 


\section{Introducción}

Alejandra Pizarnik fue una escritora argentina (Avellaneda, 1936 - Buenos Aires, 1972). Su construcción poética y sus diarios expresan el inconformismo social, de género y hacia el mismo lenguaje. Sus escritos rompen los parámetros de las décadas precedentes, no busca imitar a nadie, sino endilgar una literatura subversiva (Calahorrano, 2010). Por ello se visualiza una diferencia de construcción en su concepto de mujer, ello indicaría que cada mujer construye un concepto sobre qué es ser mujer desde su postura política, social, su nacionalidad, su raza y su historia de vida (Sales, 2017). Las diferentes interpretaciones subjetivas de ser mujer son uno de los puntos que también se visualiza en el feminismo interseccional. Desde esta línea, el término interseccionalidad aporta con construir el concepto de mujer desde lo complejo de la vida social, con una perspectiva multidisciplinaria (Sales, 2017). Desde la misma postura, Simone de Beauvoir (2011) indicó que la mujer se construye, teoría que Poulain de la Barre y Harriet Taylor corroboran. Lo que significa que no hay nada biológico que justifique la discriminación hacia las mujeres, la cultura es el medio de dónde parten los conceptos de ser mujer e incluye los estereotipos. Por ello, la mujer se encuentra envuelta en un ideal femenino, un concepto de belleza eterna, sumisión, maternidad y trabajo del hogar. $Y$ al enfrentarse a estos ideales, decide construirse diferente, dejar la postura de vasalla y volverse independiente. En este sentido, la mujer se permitirá seguir sus expectativas, desde un deseo propio y no desde un deseo impuestos por una sociedad machista (Beauvoir, 2011).

En el caso de Pizarnik, su escritura está enmarcada en el erotismo e irreverencia, quitarse las categorías de género y de transgredir cualquier concepto relacionado con el ideal femenino (Calahorrano, 2010; Sales, 2017). No obstante, por más que la autora no quiera pertenecer a ningún ismo, ni a ningún movimiento, en varios poemas se vislumbra las siguientes temáticas: 1) la noción de mujeres unidas, 2) el irse en contra de los estereotipos de género, 3) la soledad, 4) independencia de una mujer, 5) el apropiarse del cuerpo, 6) la construcción del concepto de mujer desde una perspectiva "outsider",7) las barreras infranqueables entre los derechos que posee una mujer y los del hombre, 8) el lenguaje como insuficiente en el marco de descripción sobre versiones de realidad. Asimismo, opta por la androginia en los escritos como expresión subversiva ante una elección de género de tipo heterosexual, en contextos conservadores.

Históricamente, en Argentina desde 1920 a 1940 se perseguía masivamente a la homosexualidad, el lesbianismo y diferentes orientaciones sexuales (Bruña, 2012). Pizarnik se posiciona desde la sublevación, la deconstrucción de la mujer enraizada en el patriarcado. Por ello dimana esa necesidad de generar una elección diferente y de reconstruir conceptos. De algún modo, el proceso de vida que tuvo Pizarnik es un camino que construye para cambiar lo que es ser mujer y recuperar de nuevo nuestra voz (García, 2009; Sales, 2017).

Este trabajo tiene como objetivo general describir desde la poesía, los diarios y citas de Pizarnik sobre la construcción del concepto de mujer relacionando con el feminismo interseccional. Mientras que los específicos serían: identificación de la poesía, fragmentos de diarios y citas de Pizarnik relacionadas con la construcción del concepto de mujer. 


\subsection{Marco teórico}

Para comprender los escritos de Alejandra Pizarnik es pertinente remontarnos a perspectivas teóricas que posibiliten comprender sus escritos. Así, recuperaremos elementos del feminismo interseccional para entender cómo se construye el concepto de mujer desde este enfoque.

Con respecto al origen de la interseccionalidad, Cruells (2015) expone que el término aparece en 1989 en el artículo de Kimberlé Crenshaw, denominado des marginalización interseccional de la raza y el sexo, que es una crítica al feminismo negro de la doctrina antidiscriminación. A partir de la insatisfacción hacia el feminismo clásico burgués, las mujeres afroamericanas exponen su apreciación de desigualdad, que no es la misma vivida por otros grupos, lo que implicaría que las estructuras sociales, históricas, de raza y de género, modifican el tipo de desigualdad y problemáticas percibidas por cada grupo de mujeres.

Por más que el término aparezca en esa época de los 80 s, la problemática de la articulación de las desigualdades existía mucho antes con el feminismo sufragista y el feminismo negro. Por ello varias representantes feministas tales como Bell Hooks, Moraga, Anzalfúa, Spelman, Lugones, Ángela Davis y otras, analizaron las experiencias de desigualdad social y política en diversos grupos. A su vez, manifestaron preocupación ante las desigualdades en marcos de raza, orientación sexual, condición social, nacionalidad y otras variables (Cruells, 2015).

Adicionalmente, Expósito (2012) indicó que la construcción como mujer es denominada como "compleja", en el sentido de que interactúan dinámicamente varios factores. Los factores que se encuentran inmiscuidos en el ser mujer son: el contexto, raza, historia, nacionalidad, el grupo de pertenencia, la discriminación experimentada por el grupo, experiencias, familia, el nivel socioeconómico, entre otros. Cada grupo social tiene una concepción de ser mujer; por consiguiente, la interseccionalidad vendría a ser un enfoque que capta la complejidad de ser mujer en el marco del análisis de prejuicios y discriminación, que cada grupo percibe con objetivos de concientizar y cambiar las prácticas sociales hacia una política de derechos y equidad.

La perspectiva interseccional permite comprender, en primer lugar, las diferentes relaciones sociales establecidas entre grupos. En segundo, los espacios sociales con la finalidad de generar análisis y reflexiones sobre los recursos de las administraciones, para lo que se observará si hay un trámite y potencialización de la igualdad o la desigualdad. En tercer lugar, identifica las desigualdades, prejuicios y discriminaciones, realizando un proceso de síntesis. Finalmente, se encamina el objetivo primordial, asociado a realizar prácticas de igualdad del trato y de derechos (Expósito, 2012).

Con el mismo enfoque, McCall (2005) agrega que la interseccionalidad capta a la construcción de ser mujer desde factores multidisciplinarios. Mediante el análisis de grupos, el enfoque busca que la mujer elija su manera de conceptualizarse, defendiendo sus derechos, asimismo, accediendo a oportunidades y beneficios igualitarios, tomando en cuenta que existe desigualdad entre grupos de mujeres. 
En este sentido, el feminismo interseccional se basa en un continuum, primero se ubica el enfoque anti categorial, posteriormente, se sitúa en el enfoque intra-categorial y finalmente, en el inter-categorial. En la fase del enfoque anti-categorial, el feminismo interseccional no simplifica la realidad social en categorías, sino que se enfoca en la crítica de la desigualdad, injusticias, prejuicios y discriminaciones existentes en general. En la siguiente fase, el enfoque intra-categorial deconstruye los grupos, incluyendo los no visibles o excluidos. Posteriormente se centra en el análisis de la interacción de endogrupos, prestando atención en la discriminación y la desigualdad existente para abolirla o generar actividades que aporten con el cambio. Finalmente, la fase del enfoque inter-categorial constata la existencia de relaciones de desigualdad entre distintos grupos sociales o exogrupos (McCall, 2005).

Adicionalmente, Vigoya (2016) indicó que las categorías que se analizan de manera teórica y práctica. Además, expresa que hay seis presupuestos básicos en virtud de analizar situaciones concretas y específicas desde el enfoque interseccional. Los presupuestos son los siguientes: a) existe más de una categoría de diferencia en todo problema y proceso político que está implicada, b) atender a todas las categorías es primordial, pero las relaciones entre categorías son netamente empíricas, c) cada categoría es diversa en sus interior, d) las categorías diferente son conceptualizadas desde la perspectiva de factores dinámicos de orden individual e institucional, los mismos estarán sujetos a cuestionamientos, e) la investigación interseccional examina, analiza e interroga las categorías y los factores que interactúan en las mismas, f) la interseccionalidad requiere procesos desde marcos teóricos como empíricos.

El feminismo interseccional critica las concepciones de identidad grupal, reduccionistas por parte de prácticas y estrategias políticas. No busca con ello anular las categorías de la identidad, al contrario, busca volverlas más complejas e incluyentes. Eso implica que los grupos desaventajados empiezan a empoderarse, a tomar espacios en la agenda pública y a demandar igualdad de derechos (Cruells, 2015).

\section{Antecedentes}

En el tema de análisis de la obra de Pizarnik existen varios aportes (Acosta \& Rodríguez, 2017; Flores, 2017; Torres 2004). A pesar de la importancia de los abordajes, es relevante también poder relacionar la obra con el enfoque del feminismo interseccional. Siendo el enfoque un marco de comprensión de la construcción de la mujer incluyente y que genera conciencia sobre los mecanismos de prejuicios y discriminación hacia ciertos grupos.

Entorno a estudios vinculados a análisis de escritos de Alejandra Pizarnik, Flores (2017) indicó que en los diarios de la poetisa se evidencia: un inconformismo con su patria, una frustración por la insuficiencia del lenguaje, trata de deshacer su identidad, no perteneciendo a categorías, inconformismo en roles de género y destrucción con conceptos de género establecidos. Pizarnik tuvo un proceso de construcción prolongado y complejo, con un fuerte rechazo a imposiciones externas. A su vez, transgredió cualquier norma social acorde con la desigualdad. Se reconoce que por más que la escritora no desea encasillarse en grupos, fue una mujer que luchó por la emancipación de roles de género. 
En consonancia, Torres (2004) sostuvo que Pizarnik fue la mejor poetisa de Latinoamérica y su obra expresa la máxima libertad que puede tener un ser humano. En su análisis, tanto de la poesía completa, como de los diarios, percibe varios temas, inicialmente, la historia de Pizarnik como una niña tímida y solitaria. Luego se denota el sentimiento de su patria como ajena. En tercer lugar, su exilio a Francia. En cuarto lugar, su inconformismo con las políticas de género y la imposición social. En quinto lugar, su apropiación del cuerpo y sus deseos. Finalmente, su angustia y tristeza profunda que subyace en cada escrito. La poetisa trató de deconstruir cualquier imposición social, ser auténtica e independiente, quizás esa revolución que quería ejercer no fue suficiente para sostener su vida. No obstante, cabe recalcar que Pizarnik realiza grandes aportes y es un ícono de revolución.

En otra investigación, Acosta \& Rodríguez (2017) realizaron un análisis de la poesía de Pizarnik, evidenciando que los temas recurrentes son: el exilio, el liberarse de cualquier categoría, el ser outsider o el ser marginada, el no querer pertenecer a ningún grupo, ni lugar y el deseo de no seguir la imposición de género. En conclusión, siguiendo la línea de estudio, la autora tiene una vida independiente, donde lo primordial es escuchar los deseos del cuerpo y los sentimientos desligados a la conservación de la especie y al rol de mujer ortodoxo.

Desde la misma línea, Martínez (2011) en su análisis de diarios sostuvo que Pizarnik se reveló contra los roles de género, en los cuales la mujer era un sujeto que tenía que ser solamente responsable del hogar, con una orientación de preferencia heterosexual y para beneficio de la sociedad machista debía seguir un deseo y una femineidad impuesta. A partir de la lucha contra la imposición social Pizarnik construyó un yo independiente e insatisfecho.

Según Calahorrano (2010), Pizarnik escribe una poesía erótica, dónde el cuerpo y los instintos son aceptados como parte de vivir, lo que tiene una connotación feminista y subversiva para el contexto. La autora analiza poemas y fragmentos de diarios, evidenciando que Pizarnik, más allá del género, produce una escritura donde defiende la integridad humana, busca una nueva legitimidad en la palabra de las mujeres. Otro de los temas frecuentes es el cuerpo y su conducción hacia éxtasis como parte de vivir eligiendo el deseo, porque parte de la transgresión de los roles de género es dar cuenta de que una mujer puede tener un cuerpo del cuál puede disfrutar.

En este mismo sentido, García (2009) indicó que Pizarnik es una poetisa que transita un recorrido de vida hacia la recuperación de la voz femenina, de libertad. En sus escritos se visualiza la autonomía, la cual se alcanza con perseverancia, relata las maneras del devenir de la independencia de las mujeres. Además, se encuentra en un proceso constante de construcción de conceptos y se denota en su lenguaje. Otro de los temas recurrentes es el del deseo propio, para construirnos como mujeres es necesario conocer lo que queremos, profundizar en el desarrollo y conocimiento personal como inicio de la crítica a la imposición social.

En síntesis, a pesar de que varios autores/as (Acosta \& Rodríguez, 2017; Calahorrano, 2010; Flores, 2017; García, 2009; Martínez, 2011; Torres, 2004) han analizado a Alejandra Pizarnik; no obstante, en el presente aporte se pretende unificarlo con el feminismo interseccional. 


\section{Metodología}

Se realizó una investigación cualitativa, con alcance descriptivo (Montero \& León, 2007). Estos estudios se interesan en describir una situación o concepto, además, busca profundizar en el tema a abordar, que sería un análisis de la poesía de Pizarnik hacia la construcción de la mujer en el feminismo interseccional (Montero \& León, 2007).

El investigador recolecta datos sobre historias de vida y experiencias de personas o autores/as, con el objetivo de describir y analizar materiales escritos o discursivos (Hernández et al., 2006). Según Aguilera (2016), el diseño narrativo es una herramienta que permite analizar información con finalidades de entender su simbología y sus contextos. Desde este diseño se posibilita el describir patrones de conducta, actitudes, habilidades, ideología, contexto y conexión con la teoría.

Desde la misma línea, Feijóo et al. (2019) sostuvo que en el diseño narrativo el lenguaje es fundamental en la interacción entre un sujeto y su contexto, integrar los elementos que posee el lenguaje, tanto oral, como escrito, lo que proporciona recursos lingüísticos que facilitan la representación, estructuración, profundización y comprensión del relato.

Se recolecta información a partir de fuentes bibliográficas que aportarán con la descripción y el análisis de poesía de Pizarnik (Hernández et al., 2006).

\section{Resultados}

Alejandra Pizarnik fue poetisa, escritora y traductora Argentina (1939-1972), hija de inmigrantes judíos de origen polaco. Estudió filosofía en la universidad de Buenos Aires, Argentina. Asimismo, estaba arraigada a la pintura, por ello se graduó en Juan Batlle Planas. Fue una persona que no estaba conforme con lo que sucedía en contextos de Argentina. Remontándonos a los años 2040, los grupos conservadores de marcada mentalidad católica no podían tolerar orientaciones sexuales distintas a la heterosexual. Consecuentemente, las persecuciones masivas por parte de las actuaciones policiales fueron frecuentes hacia personas con distintas orientaciones sexuales, tales como homosexuales, lesbianas, transgéneros, travestis entre otros grupos (Flores, 2017). Posteriormente, desde los 1960 al 1964 vivió en París, Francia, trabajó para la revista cuadernos y tradujo a Anthony Artaud, Henry Michaux, Yves Boneffoy entre otros/a autores/as, asimismo, tradujo ensayos de la Universidad Sorbona (Torres, 2004).

Su poesía anegaba en un pensamiento de lucha contra la imposición social, se basó en el cuestionamiento de lo establecido, entre ellos, en temas relacionados al género, a lo que es ser mujer y a la crítica del lenguaje como herramienta insuficiente para expresar una realidad interna y externa. Adicionalmente, Pizarnik prefirió no inclinarse a alguna orientación sexual, ello se visualizará en sus diarios y escritos como androginia, término acuñado característico de una persona que está constituida por una parte masculina y otra femenina. El propósito que tenía la autora era el de abolir los estereotipos en los roles de género, aunque subyace el ideal de totalidad (Flores, 2017). 
Ello se evidencia en el siguiente poema:

"Roto marco centra este todo

de árbol castrado llorando

medir cada paso a lo largo

si no se perturba la luna

la luz rodea blancuras

de nabos rallados...

girar girar girar percibir junto al marco roto

sentires de tacos y muelas

querer agarrarlo todo" (Pizarnik, 2000, p. 28).

Su vida fue una revolución, anti sistema, anti pertenecer a un ismo, anti rol de género; no obstante, nos deja una construcción de la palabra mujer que destruye el femenino ideal explicado prolijamente por Simone de Beauvoir (Flores, 2017). Pizarnik tuvo una mente brillante, la cual le produjo estragos y dificultades mentales. Su amiga María Elena Arias López había percibido la depresión aguda de Pizarnik que le llevaría al suicidio, por causa de una sobredosis de barbitúricos el 25 de septiembre de 1972 a los 36 años (Torres, 2004).

Recuperando información sobre poemas, citas y diarios de Alejandra Pizarnik, se encontrará los pilares en la construcción del concepto de mujer y en el feminismo interseccional. Según Sales (2017), cada mujer se construye a partir de sus experiencias, edad, raza, historia, entre otros factores sociales. Ello se integra en el feminismo interseccional que tiene como principal objetivo el respeto de diferencias en cada concepto de mujer. Ello se puede evidenciar en una frase de Pizarnik en la que pregona la unificación de grupos:

"Soy mujer. Y un entrañable calor me abriga cuando el mundo me golpea. Es el calor de las otras mujeres, de aquellas que hicieron de la vida este rincón sensible, luchador, de piel suave y corazón guerrero" (Pizarnik (s.f) citado por desorbita, 2017).

Pizarnik busca transgredir la imposición social de género. Uno de los puntos a retomar es la transgresión de la "moral" y los estereotipos de género. Existe un deseo de ruptura sobre los conceptos imperativos de la mujer netamente conservadora, sin placer, desligada del cuerpo, siempre a merced del esposo y el hogar (Flores, 2017). Así se puede observar en el siguiente fragmento de diarios de Alejandra Pizarnik:

"Primero, reconocer el mundo, lo utilitario; la previsión del futuro; las

prohibiciones morales. Luego, transgredirlas" (Pizarnik, 2013, p. 708).

A su vez, según Flores (2017), en varios fragmentos de diarios de Pizarnik la concepción de que la sociedad determina la identidad de género es predominante. Lo que significa que la configuración 
de género se encuentra en manos de otras entidades tales como, el Gobierno, la sociedad y a la cultura, más no en una misma. En función de ello, visualizamos cómo Pizarnik intenta transgredir con lo impuesto socialmente, especialmente con los estereotipos de género. Así se evidencia claramente en el siguiente fragmento:

"La ropa femenina es muy molesta. Tan ceñida e incómoda. No hay libertad para moverse, para correr, para nada. El hombre más humilde camina y parece el rey del universo. La mujer más ataviada camina y semeja un objeto que se usa los domingos. Además, hay leyes para la velocidad del paso. Si yo camino lentamente mirando las esculturas de las viejas casas (cosa que aprendí a mirar) o el cielo o los rostros de los que pasan junto a mí, siento que atento contra algo. Me siguen, me hablan o me miran con asombro y reproche. Sí. La mujer tiene que caminar apurada indicando que su camino tiene un fin. De lo contrario es una prostituta (hay también un "fin") o una loca o una extravagante. Si ocurre algo, alguna aglomeración o un choque, y me acerco, compruebo que no hay una sola mujer. Hombres. Nada más que hombres" (Pizarnik, 2013, p. 161).

Una parte de la ruptura sobre la imposición social es la de percibir a la mujer como un sujeto libre, que puede elegir casarse o no, elegir ser madre o no. En esta línea predomina ese proceso de conocer el deseo de sí misma. En los escritos de diarios de Pizarnik se acepta el lado salvaje de la mujer que corre con los lobos. Ese posicionamiento en dónde la naturaleza, la intuición y el vitalismo es una parte que la mujer no puede rechazar (Pinkola, 2001). Asimismo, expresa una orientación sexual distinta a la heterosexual, porque ella es la que elige su experiencia de vida sexual y sentimental. Al respecto, en el siguiente fragmento se evidenciará esta construcción de mujer diferente a la demanda social:

"Sólo quiero beber, comer y hacer el amor. [...] Tengo hambre de placeres físicos. [...] L. ya no dice que me ama, sólo me desea. Insiste en la boda. ¿Para qué? [...]. Debo dejarlo (¿Pero si me caso con él???) [...] Lo dejaré. [...] Aún en estos momentos en que me siento tan animal, tan frívola, siento firmemente que deseo estudiar, escribir, curarme, viajar, y no casarme nunca (quiero agregar que deseo alguna experiencia sexual, jwith womens!" (Pizarnik, 2003, p.129).

Según Beauvoir (2011), es servidumbre que la mujer se encuentre a merced del indumento, el maquillaje y las faenas del hogar. Por esta parte, la mujer es más que su apariencia, no se orienta a un camino unívoco asociado con la seducción. Esto no significa que una mujer no pueda 
arreglarse, sino que su deseo de hacerlo tendría que posicionarse sobre la imposición social. Lo mencionado se puede percibir en el siguiente fragmento:

"Yo me sentía anarquista e incendiaria (a causa de mis medias azules y de mi ropa sport que no rimaba con los muebles ni con la ropa -y las caras- de los demás). Alessandro [...] no comprendía por qué yo no quería actuar; puesto que era poeta y estaba así vestida, no podía quedarme en silencio" (Pizarnik, 2013, p.729).

Pizarnik construye su subjetividad desde el concepto de la mujer solitaria, ese regresar al origen, donde la mujer guarda relación con la naturaleza y su independencia (García, 2009; Sales, 2017). Así, encontramos aquello en el poema el origen:

"Hay que salvar al viento

los pájaros queman el viento

en los cabellos de la mujer solitaria

que regresa de la naturaleza y teje tormentos

hay que salvar al viento" (Pizarnik, 2000, p.52)

Asimismo, en los poemas "has construido tu casa" y "en la jaula del tiempo", se evidencia esa soledad, como oportunidad para construirse como una mujer que rompe con los estereotipos de género, dónde no se necesita un hombre para poder levantarse. Dibuja a la mujer liberada pregonada por Simone de Beauvoir, con un largo camino de lucha (Beauvoir, 2011). Los fragmentos son los siguientes:

"Has construido tu casa

has emplumado tus pájaros

has golpeado al viento

con tus propios huesos

has terminado sola lo que nadie comenzó” (Pizarnik, 2000, p.118).

En el poema "la jaula del tiempo" se puede leer lo siguiente:

"en la jaula del tiempo

la dormida mira sus ojos solos

el viento le trae

la tenue respuesta de las hojas" (Pizarnik, 2000, p. 138). 
Desde la misma línea, en el poema "viajera de corazón de pájaro negro", se visualiza su deseo de soledad, de viajar sin necesidad de estar acompañada en su camino. Simplemente caminar sola teniendo claro el objetivo (Sales, 2017). Así, se evidencia en el siguiente poema:

"Viajera de corazón de pájaro negro

tuya es la soledad a medianoche

tuyos los animales sabios que pueblan tu sueño

en espera de la palabra antigua

tuyo el amor y su sonido a viento roto" (Pizarnik, 2000, p. 147).

Respecto a la apropiación del cuerpo, Pizarnik muestra que el cuerpo de una mujer puede sentir placer, asimismo, nos revela una estructura física la cual nos pertenece. En este sentido, amar nuestro cuerpo es parte de la aceptación como mujeres (Bruña, 2012). Así, se evidencia en el poema "revelaciones":

"En la noche a tu lado

Las palabras son claves, son llaves.

El deseo de morir es rey.

Que tu cuerpo sea siempre

un amado espacio de revelaciones" (Pizarnik,2000, p. 156).

A su vez, en diarios expone también esa necesidad de aceptar su rostro y descubrirse. Parte de esa aceptación hacia una misma, es también lograr ser auténtica. En sus diarios deviene un espacio de reflexión en dónde el ser auténtico se contrapone a lo impuesto (Martínez, 2011). Ello se evidencia en el siguiente fragmento:

"Me compré un espejo muy grande. Me contemplé y descubrí que el rostro que yo debería tener está detrás -aprisionado- del que tengo. Todos mis esfuerzos han de tender a salvar mi auténtico rostro. Para ello es menester una vasta tarea física y espiritual" (Pizarnik, 2003, p.130).

De igual manera, en el poema "texto de sombra", Pizarnik acepta su modo de ser y expresarse, como parte de su conducta revolucionaria. Brinda valor a su construcción como mujer y a la expresión que tiene en el entorno social. El uso de la voz es parte de la aceptación y del cambio con la imposición social (Martínez, 2011; García, 2009). Así, se puede leer en el siguiente poema:

"Quiero existir más allá de mí misma: con los parecidos. Quiero existir como lo que soy: una idea fija. Quiero ladrar, no alabar al silencio del espacio al se nace" (Pizarnik, 2000, p. 409). 
Asimismo, en el siguiente poema:

"Cuando vea los ojos que tengo en los míos tatuados" (Pizarnik, 2000, p. 121).

Respecto a la voz como necesaria, en el poema "nadie me conoce yo hablo la noche" se evidencia la importancia de la expresión y la necesidad de las palabras en la comprensión del mundo (García, 2009):

"Nadie me conoce yo hablo la noche

Nadie me conoce yo hablo mi cuerpo

Nadie me conoce yo hablo la lluvia

Nadie me conoce yo hablo los muertos" (Pizarnik, 2000, p. 379)

En ciertos poemas describe partes el cuerpo que no tocan nada de lo erótico, desmitificando las partes del cuerpo femenino en conceptos de perfeccionismo absoluto. De este modo se apropia de los detalles con sus características imperfectas, sujetas al tiempo (Bruña, 2012). Ello se evidencia en el poema "vagar en lo opaco", que es el siguiente:

"mis pupilas negras sin ineluctables chispitas

mis pupilas grandes polen lleno de abejas

mis pupilas redondas disco rayado

mis pupilas graves sin quiebro absoluto

mis pupilas rectas sin gesto innato

mis pupilas llenas pozo bien oliente

mis pupilas coloreadas agua definida

mis pupilas sensibles rigidez de lo desconocido

mis pupilas salientes callejón preciso

mis pupilas terrestres remedos cielinos

mis pupilas oscuras piedras caídas" (Pizarnik,2000, p. 18).

Dentro de los temas abordados por Pizarnik están el sexo, el cuerpo y el deseo propio. El problema es que la tradición del discurso patriarcal reduce el camino de la mujer a ser madre y tener hijos/as. En ello se sitúa el instinto de conservación de la especie, excluyendo la elección. Consecuentemente, Pizarnik con sus escritos intenta recuperar el cuerpo y el deseo de las mujeres con objetivo de placer y satisfacción, fuera de la imposición social (Acosta \& Rodríguez, 2017, Martínez, 2011, Calahorrano, 2010). En el siguiente fragmento de poema el deseo de la palabra se evidencia aquello: 
"En la cima de la alegría he declarado acerca de una música jamás oída ¿Y qué? Ojalá pudiera vivir solamente en éxtasis, haciendo el cuerpo del poema con mi cuerpo, rescatando cada frase con mis días y mis semanas, infundiéndole al poema mi soplo a medida que cada letra de cada palabra haya sido sacrificada en las ceremonias del vivir" (Pizarnik, 2020, p. 269).

Respecto a su insatisfacción con el lenguaje, es parte del inconformismo hacia la vida en la obra de Pizarnik. Su proyecto ambicioso fue el que las palabras puedan abordar la realidad, finalmente, falló en su anhelo al darse cuenta que el lenguaje expresa algo de lo que piensa y vive. Por tanto, la construcción del concepto de mujer está resguardada por la versión de realidad de cada persona, la cual puede modificarse y jamás será capaz de abarcar la realidad en sí misma. Nuestra construcción es una interpretación adaptada a nuestros esquemas mentales en interacción constante con los símbolos y discursos sociales (Flores, 2017). En el siguiente fragmento de diarios encontraremos esta insatisfacción:

"No puedes con el lenguaje. El lenguaje no puede por ti. Palabras. Todo lo que me dieron. Mi herencia. Mi condena. Pedir que la revoquen. Pedirlo con palabras. Las palabras son mi ausencia, en mí hay una ausencia hecha de lenguaje (Pizarnik, 2013, p. 1013).

A su vez, en el poema "reminiscencias" se evidencia la falta de palabras y la diferencia de discursos:

"Dos gargantas raspadas

dos besos comunicantes de la visión de

dos promesas gimientes de

Tremendas locuacidades lejanas

dos promesas de no ser de sí ser de no ser

dos sueños jugando la ronda del sino en

derredor de un cosmos de

champagne amarillo blanquecino

dos miradas cerciorando la avidez de una

estrella chiquita" (Pizarnik, 2000, p.13).

De acuerdo con Martínez (2011), Pizarnik optó por expresar su existencia a través de las palabras, aunque de acuerdo a la autora no eran suficientes para abordar el deseo o para explicar su postura de no pertenencia a nada. Entre ese deseo de no pertenencia, sitúa su discurso en la 
incertidumbre. En el siguiente fragmento del poema "cenizas" visualizamos lo significativo de las palabras:

"Hemos dicho palabras,

Palabras para despertar muertos,

Palabras para hacer un fuego,

Palabras dónde poder sentarnos y sonreír.

Hemos creado el sermón del pájaro y del mar,

El sermón del agua,

El sermón del amor.

Nos hemos arrodillado y adorado frases extensas

Como el suspiro de la estrella,

Frases como olas,

Frases con alas" (Pizarnik, 2000, p. 82)

Hasta aquí se han presentado los hallazgos de la presente investigación, en función de los objetivos específicos del estudio. Lo que se propone también es relacionar los conceptos de mujer anteriormente mencionados a la experiencia interseccional, concepto retomado por Crenshaw en 1989. Ella mencionó que cuando hablamos de feminismo es necesario reconocer las distintas necesidades y experiencias de todas las mujeres y defender las alianzas como base de la organización. De esta manera, en Pizarnik, por más que no quiera pertenecer a ningún grupo, ha corroborado con temas sobre la diferencia entre cada mujer, la unificación hacia la lucha y el apoyo. Cada mujer escoge un camino y su construcción a través de su historia, la idea base del feminismo interseccional concientizarnos y modificar prácticas discriminatorias hacia grupos oprimidos o grupos en los cuáles no pertenecemos (exogrupos) (Sales, 2017).

En 1960 varios grupos de mujeres empiezan a comentar acerca de su cansancio sobre un feminismo representado únicamente por una mujer blanca occidental, heterosexual y burguesa. Cuándo la categoría "mujer" es compleja, porque no solo es diferente en cada grupo social, sino que cambia en cada mujer. Es en ese momento en que la interseccionalidad trata de captar la complejidad de la mujer desde perspectivas multidisciplinarias. Este nuevo enfoque se entiende desde un continuum que inicia con el enfoque anti-categorial, intra-categorial hasta la intercategorial (McCall, 2005).

Inicialemnte, el enfoque anti-categorial se basa en mostrar que las categorías simplifican la realidad social. Por ello critica las injusticias o desigualdades, intenta destruir los privilegios de determinadas realidades y relaciones sociales. En segundo lugar, el enfoque intra-categorial parte de la deconstrucción crítica de las categorías sociales, enfocándose en grupos no visibles o excluidos. Trata de analizar la dinámica de estos individuos, buscando concientizar, modificar prejuicios y discriminación. Finalmente, el enfoque inter-categorial, que constata la existencia de 
relaciones de desigualdad entre grupos sociales, se focaliza sobre distribuciones desiguales de recursos, tanto simbólicos, como materiales. Así se evidencia desigualdad en recursos, salarios y oportunidades. Con este contínuum pretende ser consciente de las diferencias para intentar modificar conductas discriminatorias, integrar y luchar por derechos igualitarios para todas. A su vez, respetar el deseo de cada mujer por la razón de que cada una se construye a partir de la interacción de múltiples factores (McCall, 2005).

El término interseccional se formuló como respuesta al feminismo transversal que no consideraba mujeres de razas, condiciones de vida y clases sociales diferentes. Desde este enfoque es posible identificar las desigualdades sobre las mujeres, asimismo, sobre distintos grupos específicos. Ello nos permite comprender las diferentes relaciones sociales establecidas entre grupos y los espacios sociales analizando a profundidad si los recursos de las administraciones potencian la igualdad o la desigualdad. Es a partir de la identificación de desigualdades y discriminaciones múltiples que se encamina el objetivo primordial, asociado a realizar prácticas de igualdad de trato y de derechos (Expósito, 2012).

Cruells (2015) acotó que la interseccionalidad permite volver explícitas y nombrar las desigualdades en juego y las relaciones de desigualdad con otros factores. En relación de políticas de igualdad de género, se evidencia mayor desigualdad asociada a la orientación sexual, la edad, raza, diversidad funcional, clase, salud y origen. Sobresale por la existencia de la amplitud de desigualdades, por tanto, con el enfoque interseccional se incide en futuras acciones y prácticas.

Con lo anteriormente mencionado, la poesía, frases y diarios de Pizarnik son aportes pertinentes. Por su parte, muestra una visión de mujer amplia, la ruptura con los roles de género, estereotipos, diferencias que pueden unificar si existe respeto para cada una de las construcciones discursivas y lucha por los derechos. De igual manera, mediante la desconstrucción se puede entender las diferencias que nos unifican, nuestra postura social, política, laboral, personal y discursiva. Quizá encontremos unas vetas en común sobre luchas y derechos, la idea base es mantenernos juntas como mujeres.

\section{Discusión}

Varios/as autores/as (Acosta y Rodríguez, Calahorrano, 2010 2017; Bruña, 2012; Flores, 2017; García, 2009; Sales, 2017; Torres, 2004) han analizado escritos de Alejandra Pizarnik con aportes sobre conceptos de la construcción de mujer y el feminismo interseccional. No obstante, Pizarnik no se categorizaba en ningún grupo, prefería estar fuera de cualquier etiqueta como parte de su fidelidad a lo que ella llamaba el ser auténtica. Según Martínez (2011), la escritora en varios fragmentos de sus diarios expresa una distancia respecto a las mujeres feministas. Ello se centra en su concepto liberal de decidir ser anti todo, aun así, en varios escritos expuso idea de la trasgresión de los roles de género impuesto, la emancipación femenina y el deseo ante todas las cosas. Además, indicó que la unión es lo necesario y el simple hecho de aportar como lo hizo, analizar y polemizar lo impuesto es un acto de emancipación y revolución. En sus obras, su voz como mujer luchadora y crítica resuena hasta la actualidad. 
Pizarnik prefirió no inclinarse a alguna orientación sexual, asimismo, a ningún movimiento que implica un sentido de pertenencia. Ello se evidencia en su androginia en los escritos, con el propósito que tenía la autora era el de abolir los estereotipos en los roles de género, no determinarse ni como mujer, lesbiana o bisexual, simplemente demandar cambios en una sociedad que para ese tiempo impedía el libre albedrío, tanto en objetivos de vida, orientación sexual y expresión literaria (Flores, 2017). Si bien la postura de Pizarnik respecto al género y la sexualidad desafía cualquier encasillamiento, es factible evidenciar en ciertos fragmentos aportes hacia el concepto de mujer y a la unificación de grupos.

Las críticas a la interseccionalidad son varias (Salem, 2015; Carbin \& Edenheim, 2013), sobre todo enfocadas en el feminismo interseccional como una utopía, la acusación de haberse convertido en un lenguaje común o en una perspectiva sumamente inclusiva, pueden ser acertadas. No obstante, el enfoque interseccional ha permitido que cada persona pueda concientizarse de sus propios mecanismos de discriminación y prejuicios, para de ese modo aceptar la existencia de varios feminismos y situaciones diferentes entre otros grupos de mujeres (García, 2009; Sales, 2017; Torres, 2004).

La interseccionalidad ha generado debates entre el feminismo y las reflexiones académicas. Primero el género se sitúa como eje primordial en la regulación de las políticas de igualdad y se considera la heterogeneidad de las situaciones de varios grupos de mujeres. Si bien el enfoque ha sido catalogado como insuficiente para incluir a todos los grupos, la interseccionalidad es una herramienta útil en el análisis exacto de los niveles relacionados a la desigualdad en función de múltiples variables en determinados grupos. Es a partir de los análisis de diversidad que el enfoque permite actuar desde la equidad (Expósito, 2012; Sales, 2017).

La dificultad que se debate del feminismo interseccional es el método de fragmentación, porque puede excluir a grupos sociales con realidades más complejas. Lo que significa que por más que se focalice en grupos excluyentes, podrá haber otros grupos que no se tomen en cuenta. Como el método es costoso y difícil de aplicar, habrá dificultad en incluir la infinidad de grupos. Dentro de esta línea, el trabajo en las administraciones públicas se vuelve inviable en su aplicación. Por ello se trabaja a través de aproximaciones a la interseccionalidad (Expósito, 2012).

En cuanto a las aproximaciones, la interseccionalidad posee un diagnóstico eficaz en cuanto a desigualdad, prejuicio y discriminación. El enfoque analiza y valora las situaciones particulares que puedan ser significativas en la desigualdad social. Puede englobar información que aporte con el cambio y potencialice las prácticas de equidad. A su vez, determina que las desigualdades no son equiparables entre sí, por lo tanto, no pueden tener un mismo tratamiento (McCall, 2005; Expósito, 2012; Sales, 2017; Torres, 2004).

Según Vigoya (2016), otros debates del feminismo interseccional sobre niveles macro y micros son los siguientes: por un lado, está la idea de que los análisis desde el enfoque se han vuelto excesivamente introspectivos, reduciéndose al análisis de narraciones de las identidades. Por otro lado, hay la percepción de un análisis deteriorado con respecto a las dimensiones subjetivas de las relaciones de poder, lo que remontará a la falta de teorización de temas sobre el poder. A pesar de que al inicio del feminismo interseccional fue juzgado como conflictivo y amenazante para el 
feminismo "blanco" y otros reduccionistas, pasó a convertirse en una de las teorías del feminismo aceptada en la actualidad. Así, desde el feminismo interseccional se ha trabajado en las múltiples dimensiones de análisis de grupos (Vigoya, 2016).

Se ha logrado describir el debate existente entre los escritos de Pizarnik en la construcción de conceptos de mujer y el feminismo interseccional. Asimismo, entreteje la importancia de los conceptos y temáticas de Pizarnik con el feminismo interseccional.

\section{Conclusiones}

En el actual estudio se describe la perspectiva de Pizarnik desde varias temáticas: la mujer independiente, emancipada, los roles de género, la ruptura con las imposiciones sociales, la capacidad de desear por nosotras, la necesidad de clarificar nuestro deseo y nuestro concepto de ser mujer. Asimismo, hay una ruptura del eterno femenino, concepto que puede estar en el discurso de cualquier persona. Desde este discurso, la mujer tiene las finalidades de vida enfocadas en el matrimonio, el hogar y el cuidado de hijos/as. Según Pizarnik, este objetivo de vida es diferente porque la mujer para poder emanciparse necesita escuchar su deseo propio y puede elegir una vida diferente a la imposición social.

En los escritos de Alejandra Pizarnik se denota la habilidad de generar nuevos lenguajes, nuevos conceptos desarraigados de la imposición social. La mujer es libre, independiente, con criterio formado para poder cuestionar y transgredir las imposiciones sociales. Además, mediante el lenguaje escrito, Pizarnik es la voz de la lucha por una política de derechos. Eso se denota en sus obras, en donde abiertamente crítica al contexto represivo, al discurso machista de una manera libre y sin miedo.

Otro de los aportes es la unificación mediante la aceptación de diferencias, no hace falta pertenecer formalmente a un movimiento, sino el aceptar las diferencias que tiene cada mujer y apoyarnos. En este sentido, en el feminismo de Crenshaw, entre mujeres existe discriminación y prejuicio hacia grupos de no pertenencia y grupos excluidos. En primer lugar, la base del enfoque interseccional es analizar y destruir conceptos con la finalidad de analizar factores multidisciplinarios. Ello implica deshilvanar esas construcciones que hemos generado hacia otros grupos para encontrar las razones de cavar un abismo infranqueable entre grupos.

El enfoque nos llama a concientizarnos, analizar y cambiar nuestros modos de pensar, sentir y actuar respecto a diferentes mujeres y grupos. Además, el considerar que el análisis de múltiples factores dentro de la dinámica de desigualdad, puede a aportar a tomar diferentes acciones y decisiones para poder trabajar en el cambio hacia los derechos de todos los grupos.

Desde la postura del feminismo interseccional, la asunción de la realidad se caracteriza por ser relacional, la vulnerabilidad de un grupo se conecta con la posición de privilegio de otro grupo o individuo. Entonces la búsqueda de unificación entre grupos se determina por la solidaridad, el respeto y la responsabilidad, es este sentido, la lucha radica en la demanda de justicia para todos los grupos frente a cualquier injusticia estructural. A su vez, se trata de una emancipación 
política hacia mejores prácticas sociales e institucionales que favorezcan nuestros derechos de ser mujeres. 


\section{Referencias}

Acosta, M., \& Rodríguez, V. (2017). La morada del lenguaje de las extranjeras: La búsqueda de un espacio otro en la poesía de Alejandra Pizarnik y Cristina Peri Rossi. Tenso diagonal, (03). https://www.tensodiagonal. org/index.php/tensodiagonal/article/view/126/107

Aguilera, F. (2016). Guía para el diseño narrativo. [Proyecto, Universidad del Desarrollo]. Repositorio Institucional UDD http://hdl.handle.net/11447/1824

Bruña, M. (2012). Pizarnik-artefacto: autoconfiguración y mito. Letral, (8). https://doi.org/10.30827/rl.v0i8.3683

Beauvoir, S. (2011). Segundo sexo. En Simone de Beauvoir (comp). De bolsillo.

Carbin, M., \& Edenheim, S. (2013). The intersectional turn in feminist theory: A dream of a common language? European Journal of Women's Studies. https://doi.org/10.1177/1350506813484723

Calahorrano, P. (2010). Cuerpo y muerte: la sexualidad que exhala Alejandra Pizarnik a través de la muerte deseada. Divergencias. Revista de estudios lingüísticos y literarios, 8(2), 92-100. https://divergencias. arizona.edu/sites/divergencias.arizona.edu/files/articles/CalahorranoPizarnik.pdf

Crenshaw, K. (1989). Demarginalizing the intersection of race and sex: A black feminist critique of antidiscrimination doctrine, feminist theory and antiracist politics. u. Chi. Legal f., 139.

Cruells, M. (2015). La interseccionalidad política tipos y factores de entrada en la agenda política, jurídica y de movimientos sociales. [Tesis doctoral, Universidad Autónoma de Barcelona]. https://www.tdx.cat/handle/10803/288224\#page $=4$

Desorbita. (08 de marzo de 2017). Dos poemas de Alejandra Pizarnik. https://desorbita.wordpress. com/2017/03/08/dos-poemas-de-alejandra-pizarnik/

Expósito, C. (2012). ¿Qué es eso de la interseccionalidad? Aproximación al tratamiento de la diversidad desde la perspectiva de género en España. Investigaciones feministas, 3. https://doi.org/10.5209/rev INFE.2012. v3.41146

Feijóo, J., Chamizo, Sa., \& Trapero, J. (2019). El diseño narrativo: un nuevo paradigma para la comunicación.

Flores, I. (2017). Identidad, género y lenguaje en la construcción poética de Alejandra Pizarnik. Tesis de pregrado. Universidad del País Vasco: España. https://addi.ehu.es/bitstream/handle/10810/21321/TFG FloresDepardieu\%2cl.pdf?sequence=2\&isAllowed=y.

García, M. (2009). Transitando por los caminos del espejo de Alejandra Pizarnik, hacia la (re)construcción de nuevas subjetividades femeninas. Clepsydra, (8), pp. 81-89. http://riull.ull.es/xmlui/handle/915/11890 
Hernández, R., Fernández, C., \& Baptista, M. (2006). Metodología de Investigación. Mc Graw Hill.

McCall, Leslie. (2005). The complexity of intersectionality Signs. Journal of Women in Culture and Society, 30(3). https://doi.org/10.1086/426800

Martínez, A. (2011). Alejandra Pizarnik: si no me scribo soy una ausencia. Itinerarios, 14 http://itinerarios.uw.edu. $\mathrm{pl} / \mathrm{wp}$-content/uploads/2014/11/07 Arregi Itin-14.pdf

Montero, I., \& León, O. (2007). Guía para nombrar los estudios de investigación en Psicología. International Journal of Clinical and Health Psychology, 7(3), 847-862.

Pinkola, C. (2001). Mujeres que corren con los lobos. LIBERDOPLEX, S.L

Pizarnik, A. (2003). Diarios. Lumen.

Pizarnik, A. (2013). Diarios. Lumen.

Pizarnik, A. (2000). Poesía completa. Penguin Random house group.

Sales, T. (2017). Repensando la interseccionalidad desde la teoría feminista. Agora: papeles de Filosofía, 36(2).

Salem, S. (2015). Feminismo islámico, interseccionalidad y decolonialidad. Ideas y valores, 64(159). https://doi. org/10.15446/ideasyvalores.v64n159.52974

Torres, C. (2004). Alejandra Pizarnik. Revista de estudios literarios de la Universidad Complutense de Madrid, (28). http://webs.ucm.es/info/especulo/numero28/alepizar.html

Vigoya, M. (2016). La interseccionalidad: una aproximación situada a la dominación. Debate Feminista, (52). https://doi.org/10.1016/j.df.2016.09.005 
Copyright (c) 2022 Rosa Estefanía Navas Espinosa



Este texto está protegido bajo una licencia internacional Creative Commons 4.0.

Usted es libre para Compartir-copiar y redistribuir el material en cualquier medio o formato

- y Adaptar el documento - remezclar, transformar y crear a partir del material-para cualquier propósito, incluso para fines comerciales, siempre que cumpla las condiciones de Atribución. Usted debe dar crédito a la obra original de manera adecuada, proporcionar un enlace a la licencia, e indicar si se han realizado cambios. Puede hacerlo en cualquier forma razonable, pero no de forma tal que sugiera que tiene el apoyo del licenciante o lo recibe por el uso que hace de la obra.

$\underline{\text { Resumen de licencia - Texto completo de la licencia }}$ 